\title{
sciendo
}

\author{
Current Issues in Pharmacy and Medical Sciences \\ Formerly ANNALES UNIVERSITATIS MARIAE CURIE-SKLODOWSKA, SECTIO DDD, PHARMACIA \\ journal homepage: http://www.curipms.umlub.pl/
}

\section{Histopathological nephrotoxic features of high oral doses of ubiquinone in rats}

\author{
Ali Ghanim Abdullah ${ }^{1}$, Ban Ismael Sedeeq $^{2}$, Marwan Saad Azzubaidi $^{3 \star}[$ \\ ${ }^{1}$ Department of Oral and Maxillofacial Surgery, College of Dentistry, Tikrit University, Iraq \\ ${ }^{2}$ Department of Basic Medical Sciences, College of Dentistry, Tikrit University, Iraq \\ ${ }^{3}$ Pharmacology Unit, Faculty of Medicine, Universiti Sultan Zainal Abidin, Kuala Terengganu, Malaysia
}

\section{ARTICLE INFO}

Received 26 March 2021

Accepted 10 May 2021

\section{Keywords:}

Co-enzyme Q10,

nephrotoxicity,

histopathology,

rats.

\begin{abstract}
Co-enzyme Q10 (Co-Q10) plays a key role in the cellular respiration for the production of ATP. The toxicity of quinones to the kidney appears to depend on variety of factors, including genetic polymorphisms and the individual's comorbidites. The aim of the present study was to assess histologically the nephrotoxic effects of 6 weeks daily oral intake of Co-Q10 in experimental animals.

Twenty-five Wistar rats weighing between $220-270 \mathrm{~g}$ were randomly divided into two groups: experimental "treated" and control "untreated" groups $(\mathrm{n}=15, \mathrm{n}=10$, respectively). The animals of the experimental group received $300 \mathrm{mg} / \mathrm{kg}$ daily dose of gelatinous capsules of Co-Q10 by oral gavage for six weeks. At the end of the study, all animals were sacrificed under general anesthesia and samples of the kidneys were excised for microscopic histopathological assessment of renal tissue using stain. The experimental group showed a range of mild to severe dilatation of Bowman's space, with a mean corpuscular diameter of $294 \pm 38 \mu \mathrm{m}$ that was significantly higher $(\mathrm{p}<0.05)$ than that of the untreated control group $208 \pm 31 \mu \mathrm{m}$. Shrinkage to complete destruction of the glomeruli was observed in the experimental group only. The long-term use of high doses of Co-Q10 had revealed a selective nephrotoxicity towards podocytes. This might be a risk factor leading to renal proximal tubular necrosis in rats and the subsequent renal function deterioration.
\end{abstract}

\section{INTRODUCTION}

Co-enzyme Q10 (Co-Q10) or ubiquinone is a fat-soluble compound composed of a benzoquinone ring with 10 isoprene side-chains. The human body naturally synthesizes Co-Q10 via mitochondrial enzymes situated in the matrix intermembrane [1]. The electron transport chain during the process of cellular respiration for the production of ATP utilizes Co-Q10 as an electron acceptor in oxidative phosphorylation, transforming products of metabolism into ATP [2].

Plasma Co-Q10 concentrations have been reported to be significantly lower in patients suffering from chronic kidney disease (CKD) with or without hemodialysis, compared to normal controls [3]. Earlier studies provided supporting evidence regarding the benefit of Co-Q10 supplementation for improving the renal function and delaying the need for dialysis in patients with CKD [4]. The proposed

\footnotetext{
* Corresponding author

e-mail: mazzubaidi@gmail.com
}

nephroprotective mechanism of Co-Q10 was based on its anti-inflammatory activities, which enables Co-Q10 to assist in the protection against renal tissue injury and renal dysfunction induced by various drugs $[5,6]$.

Despite the presence of abundant reviews and study articles supporting the benefits of Co-Q10, quinones may still eventually cause deleterious effect on the kidneys. The decisive factors of whether Co-Q10 leads to nephroprotection or nephrotoxicity are the yields produced by the "reduction reaction" and the oxidative status of the kidney. While stable, fully oxidized quinones are less reactive and their production can quench other cellular toxins such as reactive oxygen species (ROS). In contrast, partially reduced semiquinones are free radicals that can damage DNA and lead to cell death [7]. The toxicity of quinones to the kidney appears to be multifactorial, including genetic polymorphisms and the disease status of the individual.

Recently, there are studies reporting the lack of adequate evidence that supports the use of Co-Q10 supplementation 
for nephroprotection. A study done in 2012 stated that patients who were treated with Co-Q10 after the development of CKD showed no improvement in their renal function [8]. Furthermore, another study examining the efficiency of antioxidants on the initiation and progression of diabetic nephropathy revealed that antioxidants, including Co-Q10, did not have reliable effects against the progression of the disease [9]. Therefore, Madmani and colleagues concluded that there was insufficient proof backing the use of supplemental Co-Q10 in heart failure and other diseases [10]. Additionally, El Shaikh and co-researchers stated that subjecting rats to daily oral dose of $200 \mathrm{mg} / \mathrm{kg}$ for 8 days did not significantly distort the normal renal glomeruli. However, mild degeneration of epithelial lining of some renal tubules was noticed [11].

Since limited research studies so far exist to document the histological changes after a long-term administration of moderate doses of Co-Q10, the specific aim of this study was to examine the potential toxic histopathological changes in rat kidney cells after long-term administration of a high dose $(300 \mathrm{mg} / \mathrm{kg})$ of Co-Q10.

\section{MATERIALS AND METHODS}

\section{Animals}

Twenty-five Albino Wistar rats weighing between 220 g-270 g were randomly divided into two groups: 15 experimental group and 10 control group. The rats were housed in metallic cages in the animal house of veterinary college in Tikrit University. Animals were maintained at room temperature with 12 hours dark: light cycle, receiving basal food pellets and water ad-libitum. All experiments were carried out according to the National Institute of Health Guidelines for the care and use of laboratory animals and the European Council Directive on 24 November 1986 for Care and Use of Laboratory Animals (86/609/EEC), and were approved by the Local Ethics Committee (EDB\# 20-0283-1550).

The animals of experimental group received $300 \mathrm{mg} / \mathrm{kg}$ daily dose of gelatinous capsules of Co-Q10 by oral gavage for six weeks. At the end of the experiment, all animals were sacrificed and tissue samples of kidney were excised and fixed in 10\% neutral-buffered formaldehyde solution until they were embedded in paraffin wax blocks. Subsequently, 5 - $\mu \mathrm{m}$ serial sections were cut and stained with hematoxylin and eosin (H\&E). Histological observations were done for assessment of the microscopic changes using a light microscope.

\section{Counting technique}

For each slide of the control and the experimental groups, twenty microscopic fields were considered in order to estimate and count the histological parameters [12]. A calibrated stage micrometer brand (Leitz) which consists of 100-minute lines, each line equals to $10 \mu$, along with a calibrated ocular micrometer brand (Reichert) and a light microscope brand (Meiji) were used. A superimposition between the calibrated ocular micrometer and calibrated stage micrometer was performed in the following manner: at $(\times 10)$ magnification power, each line of calibrated stage micrometer equaled to (81) line of calibrated ocular micrometer, so each line of calibrated ocular micrometer equaled to $12.3 \mu \mathrm{m}$, at $(\times 40)$ magnification power, each line of calibrated ocular micrometer equaled to 3 lines of calibrated stage, so each line of ocular micrometer is equaled to $3.3 \mu \mathrm{m}$ [13].

\section{Statistical analysis}

Microsoft Excel 2016 and SPSS (version 21.0) software programs were used for data analysis. Results were expressed as means \pm SEM. Significant differences were determined by t-test.

\section{RESULTS}

Microscopic examination of kidney sections of control rats showed the normal structure of the renal corpuscles and renal tubules as revealed in Figure 1.a, b. The mean diameter of renal corpuscles of the control group was $208.4 \pm 31 \mu \mathrm{m}$.
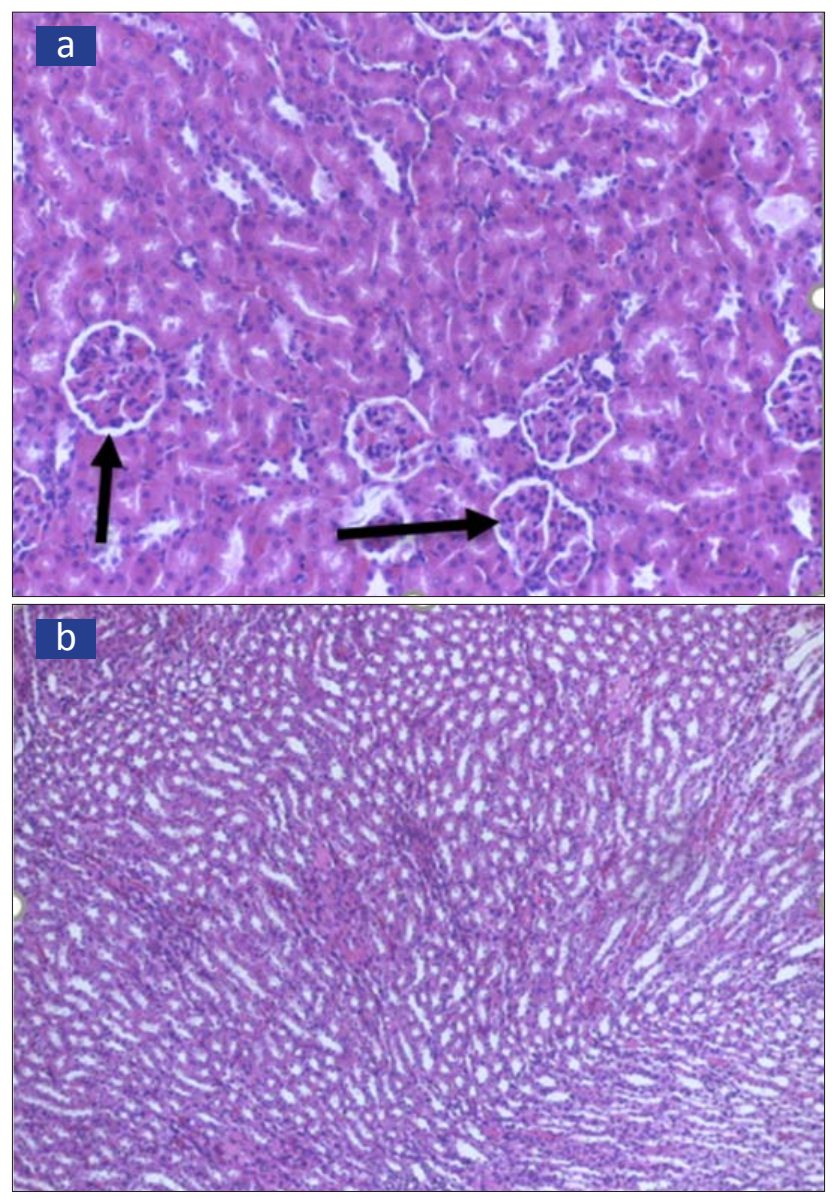

Figure 1. Depicting the control group (a) Renal cortex showing normal renal corpuscles [arrows] at $(\times 200)$ magnification power. (b) Renal tubules at $(\times 100)$ magnification power

Samples from experimental group expressed a range of mild to severe dilatation of Bowman's space, as seen in Figure 2.a with an obviously increased diameter (294.25 $38 \mu \mathrm{m})$ of renal corpuscles, which was significantly higher $(\mathrm{p}<0.05)$, than the diameter of renal corpuscle in the control group $(208.4 \pm 31 \mu \mathrm{m})$. 

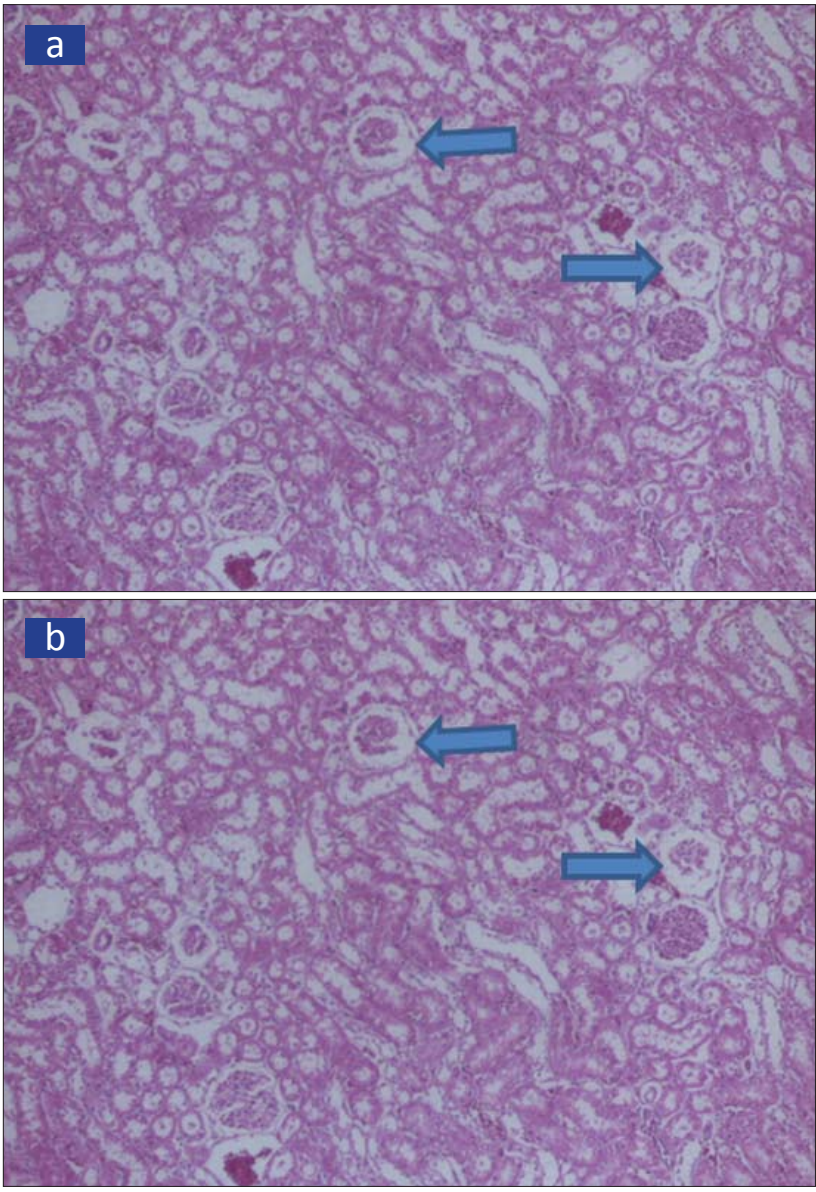

Figure 2. Depicting the experimental Co-Q10 treated group showing: (a) Widening of Bowman's space [arrows], (b) complete destruction of renal corpuscle [arrows] at $(\times 200)$ magnification power

Some sections of the experimental Co-Q10 treated group showed shrinkage to complete destruction of the glomeruli as seen in Figure 2.b, other glomeruli showed a blood-filled cavitation as observed in Figure 3.

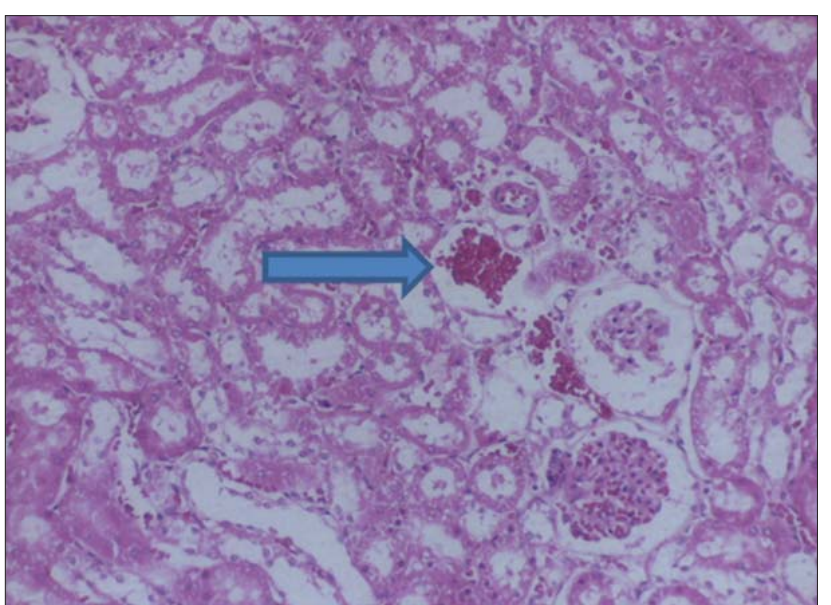

Figure 3. Depicting RBC [arrow] accumulated in the renal corpuscles of the experimental group at $(\times 200)$ magnification power

The lining of renal medullary tubules in the CoQ10-treated group appeared exfoliated, desquamated, or thin giving an impression of widening of lumen of tubules with faint cytoplasm in contrast to the normal structure of the medulla of the control group, as seen in Figure 4 ( $a$ and b).
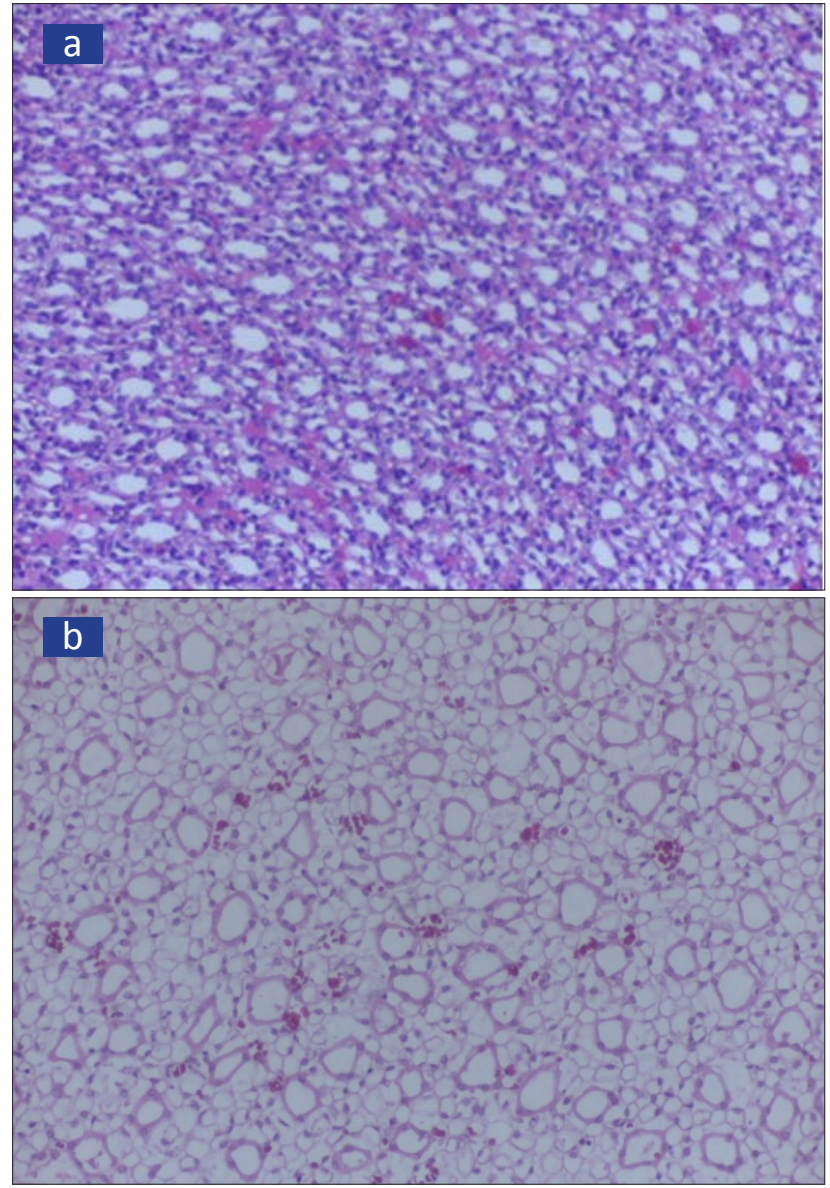

Figure 4. Medulla of the control group (a) at magnification power $(\times 100)$, experimental group $(b)$ at magnification power $(\times 200)$

\section{DISCUSSION}

Co-Q10 is an endogenously synthesized antioxidant involved in the ATP production and electron transfer chain [14]. Although the oral bioavailability of Co-Q10 is relatively low $(2.2 \%)$, the oral route is still considered since the amount needed to be absorbed by the body is considered sufficient [15]. In spite of the presence of several studies mentioning a number of medical uses of Co-Q10 [16], including its effect in minimizing precipitation of metals in kidney and the significant benefit of Co-Q10 supplementation particularly in patients with heart diseases, some studies have reported that the improvement was insignificantly trivial. A number of factors might contribute to the modest improvement of clinical conditions supplemented with Co-Q10, including subtherapeutic Co-Q10 dosage or short treatment duration and inter-individual variation in the ability to absorb Co-Q10. This can particularly lead to poor bioavailability, especially when Co-Q10 blood levels have not been determined before and after supplementation [17].

The toxicity of Quinones to the kidneys is greatly related to the degree of oxidation and reduction of products by the kidney [18]. Since excessive loading of antioxidants might have damaging outcomes to biological systems, antioxidant therapy should be carefully designed [19]. It has been reported that $200 \mathrm{mg}$ daily oral dose of ubiquinone failed to show any significant beneficial effects for hemodialytic patients [20]. The high doses of ubiquinone had revealed a selective toxicity towards podocytes, with severe renal 
proximal tubular necrosis in rats [21]. As it is well established that podocytes are the major cell of the glomerular filter, they are highly differentiated and quiescent cells involved in the pathology of numerous kidney diseases [22]. There are few, if any, studies assessing the effect of high doses of Co-Q10 on the histological structure of kidney, except for a study carried out by El Shaikh et al. in 2012 who found normal glomerular appearance with focal tubular degeneration which came in agreement with the results of the present study [11].

In contrast, no histological changes were observed by using $30 \mathrm{mg} / \mathrm{kg}$ oral doses of Co-Q10 for 14 days in a previous research [23]. With this obviously much lower dose than the dose we used in the current study, it is comprehensible that their study results were inconsistent with the outcomes of the current study results.

\section{CONCLUSION}

The most important finding peculiar to this study is that the long-term repeated use of high doses of Co-Q10 might result in a selective nephrotoxicity towards podocytes. This can be a risk factor for renal proximal tubular necrosis in rats leading to the subsequent impending renal function deterioration.

\section{LIST OF ABBREVIATIONS}

ATP - Adenosine triphosphate.

Co-Q10 - co-enzyme Q10.

CKD - Chronic kidney disease.

DNA - deoxyribonucleic acid.

H\&E - hematoxylin and eosin.

ROS - reactive oxygen species

SEM - Standard error of the mean.

SPSS - Statistical package for social sciences.

\section{ACKNOWLEDGMENT}

Authors are thankful to the associate staff in the department of basic sciences-histology at the University of Tikrit who assisted in the processing and staining of the samples slides, respectively.

\section{CONFLICT OF INTERESTS}

Authors declare that there is no conflict of interest that could affect the objectivity and credibility of the work.

\section{ORCID iDs}

Marwan Saad Azzubaidi (D)https://orcid.org/0000-0002-9118-5930

\section{REFERNCES}

1. Awad AM, Bradley MC, Fernandez-Del-Rio L, Nag A, Tsui HS, Clarke CF. Coenzyme Q10 deficiencies: pathways in yeast and humans. Essays Biochem. 2018;62:361-76.

2. Lee SQ, Tan TS, Kawamukai M, Chen ES. Cellular factories for coenzyme Q10 production. Microb Cell Fact. 2017;39:1-16.
3. Macunluoglu B, Kaya Y, Atakan A, Ari E, Kaspar C, Demir H. Serum CoQ10 levels are associated with coronary flow reserve in hemodialysis patients. Hemodial Int. 2013;17:339-45.

4. Singh RB, Kumar A, Niaz MA, Singh RG, Gujrati S, Singh VP. Randomized double blind placebo controlled trial of CoQ10 in chronic renal failure. J Nutr Environ Med. 2000;10:281-8.

5. Mehmetoglu I, Yerlikaya FH, Kurban S, Erdem SS, Tonbul Z. Oxidative stress markers in hemodialysis and peritoneal dialysis patients, including coenzyme Q10 and ischemia-modified albumin. Int J Artif Organs. 2012;35:226-32.

6. Anupama S, Pragnesh P, Nishkruti M, Pankti D. Effect of coenzyme Q10 alone and it's combination with pentoxifylline in cisplatininduced nephrotoxicity in rats. J Pharm Sci Bioscientific Res. 2016;6: 790-800.

7. Sayed-Ahmed MM, Nagi MN. Thymoquinone supplementation prevents the development of gentamicin-induced acute renal toxicity in rats. Clin Exp Pharmacol Physiol. 2007;34:399-405.

8. Tamura Y, Tanabe K, Kitagawa W, Uchida S, Schreiner GF, Johnson RJ, Nakagawa T. Nicorandil, a K(atp) channel opener, alleviates chronic renal injury by targeting podocytes and macrophages. Am J Physiol Renal Physiol. 2012;303:F339-49.

9. Ragheb A, Attia A, Eldin WS, Elbarbry F, Gazarin S, Shoker A. The protective effect of thymoquinone, an anti-oxidant and antiinflammatory agent against renal injury: a review. Saudi J Kidney Dis Transpl. 2009;20:741-52.

10. Nicolantonio JJ, Bhutani J, McCarty MF. Coenzyme Q10 for the treatment of heart failure: a review of the literature. Open Heart 2015;2:e000326.

11. El-Sheikh AA, Morsy MA, Mahmoud MM, Rifaai RA, Abdelrahman AM. Effect of coenzyme-q10 on Doxorubicin-induced nephrotoxicity in rats. Adv Pharmacol Sci. 2012;2012:981461.

12. Abdullah AG. Age related changes of submandibular salivary glands: Anatomical and histological study. MSc. Thesis; 2005

13. Al Khateeb IA. A histological study on the development of human epidermis. MSc. Thesis; 1987:52-4.

14. Littarru GP, Tiano L. Bioenergetic and antioxidant properties of coenzyme Q10: Recent developments. Mol Biotechnol. 2007;37:31-7.

15. Barakat A, Shegokar R, Dittgen M. Coenzyme Q10 oral bioavailability: effect of formulation type. J Pharm Inv. 2013;43: 431-51.

16. Al-Megrin WA, Soliman D, Kassab RB, Metwally DM, Ahmed EAM, El-Khadragy MF. Coenzyme Q10 activates the antioxidant machinery and inhibits the inflammatory and apoptotic cascades against lead acetate-induced renal injury in rats. Front Physiol. 2020;11:64.

17. López-Lluch G, Del Pozo-Cruz J, Sánchez-Cuesta A, CortésRodríguez AB, Navas P. Bioavailability of coenzyme Q10 supplements depends on carrier lipids and solubilization. Nutrition. 2019;57:133-40.

18. Alkharfy KM, Al-Daghri NM, Al-Attas OS, Alokail MS. The protective effect of thymoquinone against sepsis syndrome morbidity and mortality in mice. Int Immuno-pharmacol. 2011;11:250-4

19. Galli F, Piroddi M, Annetti C, Aisa C, Floridi E, Floridi A. Oxidative stress and reactive oxygen species. Contrib Nephrol. 2005;149:240-60.

20. Gokbel H, Gokbel H, Turk S, Okudan N, Atalay H, Belviranli M, Gaipov A, Solak Y. Effects of coenzyme Q10 supplementation on exercise performance and markers of oxidative stress in hemodialysis patients: A double-blind placebo-controlled crossover trial. Am J Ther. 2016;23:e1736-e1743.

21. Koyner JL, Sher Ali R, Murray PT. Antioxidants. Do they have a place in the prevention or therapy of acute kidney injury? Nephron Exp Nephrol. 2008;109(4):e109-17.

22. Durvasula RV, Shankland SJ. The renin-angiotensin system in glomerular podocytes: mediator of glomerulosclerosis and link to hypertensive nephropathy. Curr Hypertens Rep. 2006;8:132-8.

23. Farrag AR, Ibrahim RA, El-Sayed SN. Protective effect of Coenzyme Q10 against gentamicin induced acute renal failure in mice. J Biosci Appl Res. 2016;2:401-6. 